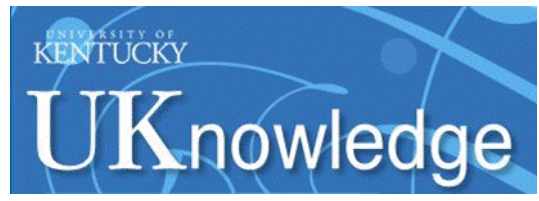

University of Kentucky

UKnowledge

$9-1952$

\title{
Convexity and Starlikeness of Analytic Functions
}

Wimberly C. Royster

University of Kentucky

Follow this and additional works at: https://uknowledge.uky.edu/math_facpub

Part of the Mathematics Commons

Right click to open a feedback form in a new tab to let us know how this document benefits you.

\section{Repository Citation}

Royster, Wimberly C., "Convexity and Starlikeness of Analytic Functions" (1952). Mathematics Faculty Publications. 5.

https://uknowledge.uky.edu/math_facpub/5

This Article is brought to you for free and open access by the Mathematics at UKnowledge. It has been accepted for inclusion in Mathematics Faculty Publications by an authorized administrator of UKnowledge. For more information, please contact UKnowledge@lsv.uky.edu. 


\section{Convexity and Starlikeness of Analytic Functions}

Digital Object Identifier (DOI)

http://dx.doi.org/10.1215/S0012-7094-52-01945-5

\section{Notes/Citation Information}

Published in Duke Mathematical Journal, v. 19, no. 3, p. 447-457.

This article is republished by permission of the copyright holder, Duke University Press. Reuse of material, except as citation, is subject to Duke University Press's permission. 


\title{
CONVEXITY AND STARLIKENESS OF ANALYTIC FUNCTIONS
}

\author{
By W. C. Royster
}

1. Introduction. Necessary and sufficient conditions for an analytic function $f(z)$ to map a circle $|z|=r$ onto a curve which is convex, or starlike with respect to a given point, are well known $[3 ; 105]$. In this paper we obtain generalizations of these conditions for certain other elementary types of curves in the $z$-plane and obtain some consequences of these generalizations.

Let $\Gamma=\Gamma(t)$ denote a directed curve $z(t)=x(t)+i y(t)$, where for $t_{0} \leq t$ $\leq t_{1}$, the real functions $x(t)$ and $y(t)$ are twice differentiable and $\left|z^{\prime}(t)\right| \neq 0$. The direction of the curve will be that generated by increasing $t$. We shall also consider one parameter families of such curves, $\Gamma=\Gamma(t, u): z=x(t, u)+i y(t, u)$, where for each fixed $u$ in $u_{0} \leq u \leq u_{1}, \Gamma(t, u)$ has the above mentioned properties for $t_{0}(u) \leq t \leq t_{1}(u)$.

Definition 1. Let $C$ be the image of $\Gamma$ under $w=f(z)$, and $\psi$ be the angle made by the tangent line to $C$ with the positive real axis. Then $f(z)$ is said to be convex on the directed curve $\Gamma$ if

$$
\frac{d \psi}{d t} \geq 0
$$

for $t_{0} \leq t \leq t_{1}$.

It will be convenient to use the notation $f(z)$ for convex functions and $F(z)$ for starlike functions.

Definition 2. Let $\phi=\arg \left(F(z)-w_{0}\right)$, and suppose that on $\Gamma, F(z) \neq w_{0}$. Then $F(z)$ is said to be starlike with respect to $w_{0}$ on the directed curve $\Gamma$ if

$$
\frac{d \phi}{d t} \geq 0
$$

for $t_{0} \leq t \leq t_{1}$.

In case $w_{0}=0$, we shall merely say " $F(z)$ is starlike on $\Gamma$." If $f(z)$ is defined in some region $R$ and the curve $\Gamma$ extends beyond this region, the statement " $f(z)$ is convex on $\Gamma$ " will mean, $f(z)$ is convex on that portion of $\Gamma$ lying in $R$.

2. The fundamental inequalities. First consider the convex case. If $\psi_{1}$ is the angle which a chord of the curve $C$ makes with the positive real axis, then

$$
\psi_{1}=\arg (f(z+\Delta z)-f(z))=\arg \frac{f(z+\Delta z)-f(z)}{\Delta z}+\arg \frac{\Delta z}{\Delta t},
$$

Received December 14, 1951; presented to the American Mathematical Society at Chicago, Illinois, April 26, 1952. The author is indebted to Professor A. W. Goodman for suggesting the topic investigated here, and for the material in $\$ 8$. 
so that in the limit

$$
\psi=\arg \frac{d z}{d t}+\arg f^{\prime}(z)=\alpha+g \log f^{\prime}(z),
$$

where $\alpha$ is the angle which the corresponding tangent to $\Gamma$ makes with the real axis. So

$$
\frac{d \psi}{d t}=\frac{d \alpha}{d t}+g \frac{f^{\prime \prime}(z)}{f^{\prime}(z)} \frac{d z}{d t}=\frac{x^{\prime} y^{\prime \prime}-y^{\prime} x^{\prime \prime}}{\left(x^{\prime}\right)^{2}+\left(y^{\prime}\right)^{2}}-\mathfrak{A}\left(i \frac{f^{\prime \prime}(z)}{f^{\prime}(z)} \frac{d z}{d t}\right) \equiv \mathfrak{C}(\Gamma, f, t) .
$$

This gives immediately

TheORem 2.1. A necessary and sufficient condition that a function $f(z)$, regular on a directed curve $\Gamma$, be convex on $\Gamma$ is that

$$
\mathcal{e}(\Gamma, f, t) \geq 0
$$

for $t_{0} \leq t \leq t_{1}$.

To obtain a criterion for starlikeness, observe that if $F(z) \neq w_{0}$, then

$$
\begin{gathered}
\phi=\arg \left(F(z)-w_{0}\right)=\mathfrak{g} \log \left(F(z)-w_{0}\right), \\
\frac{d \phi}{d t}=-\Re\left(i \frac{F^{\prime}(z)}{F(z)-w_{0}} \frac{d z}{d t}\right) \equiv s(\Gamma, F, t) .
\end{gathered}
$$

TheOREM 2.2. If $F(z) \neq w_{0}$ on $\Gamma$, and is regular on $\Gamma$, then a necessary and sufficient condition that $F(z)$ be starlike with respect to $w_{0}$ on $\Gamma$ is that

$$
s(\Gamma, F, t) \geq 0
$$

for $t_{0} \leq t \leq t_{1}$.

From these two results a generalization of Alexander's theorem [1] follows immediately.

Theorem 2.3. Let h be a complex constant and let $f(z)$ and $F(z)$ be such that on the directed curve $\Gamma$

$$
F(z)-w_{0}=h f^{\prime}(z) \frac{d z}{d t} \neq 0
$$

Then if $f(z)$ is convex on $\Gamma, F(z)$ is starlike with respect to $w_{0}$ on $\Gamma$, and conversely.

Note that not both $F(z)$ and $f(z)$ can be regular on $\Gamma$ unless $z^{\prime}(t)$ is regular on $\Gamma$. However if $\Gamma$ is an analytic curve and the parameter $t$ is suitably chosen $z^{\prime}(t)$ will be regular on $\Gamma$. Note further that if $h=-i, w_{0}=0$, and $\Gamma$ is a circle of radius $r$, then $z=\mathrm{re}^{i t}, z^{\prime}(t)=i z$ and (2.5) becomes $F(z)=z f^{\prime}(z)$. Thus Theorem 2.3 contains Alexander's theorem as a special case.

Differentiating logarithmically equation (2.5) yields

$$
\frac{F^{\prime}(z)}{F(z)-w_{0}} \frac{d z}{d t}=\frac{f^{\prime \prime}(z)}{f^{\prime}(z)} \frac{d z}{d t}+\frac{d^{2} z / d t^{2}}{d z / d t}
$$


and equating imaginary parts,

$$
\mathcal{s}(\Gamma, F, t) \equiv \mathfrak{C}(\Gamma, f, t) .
$$

From this the proof of Theorem 2.3 is obvious.

3. Ellipses. Let $\Gamma_{e}$ denote the ellipse $z=a \cos t+i b \sin t, a \geq b>0, c^{2}$ $=a^{2}-b^{2}$. Here

$$
z^{\prime}(t)=-a \sin t+i b \cos t=\left(c^{2}-z^{2}\right)^{\frac{1}{2}},
$$

where the value of the radical is determined by continuity from the condition that at $t=0, z=a, z^{\prime}(t)=b i$. Since the ellipse $\Gamma_{e}$ encloses both the foci $z= \pm c$, $\left(c^{2}-z^{2}\right)^{\frac{1}{2}}$ is single-valued on $\Gamma_{e}$. The condition for convexity of $f(z)$ on $\Gamma_{e}$ is

$$
\mathcal{C}\left(\Gamma_{e}, f, t\right) \equiv \frac{a b}{\left|c^{2}-z^{2}\right|}-\mathcal{Q}\left(i \frac{f^{\prime \prime}(z)}{f^{\prime}(z)}\left(c^{2}-z^{2}\right)^{\frac{1}{2}}\right) \geq 0
$$

and for starlikeness

$$
\S\left(\Gamma_{e}, F, t\right) \equiv-\Re\left(i \frac{F^{\prime}(z)}{F(z)}\left(c^{2}-z^{2}\right)^{\frac{1}{2}}\right) \geq 0 .
$$

Equation (2.5) with $w_{0}=0$ becomes

$$
F(z)=h f^{\prime}(z)\left(c^{2}-z^{2}\right)^{\frac{1}{2}} .
$$

If $f(z)$ is a polynomial with all its zeros inside $\Gamma_{e}$, then by the Gauss-Lucas theorem all of the zeros of $f^{\prime}(z)$ lie inside $\Gamma_{e}$. Since the zeros of $F(z)$ given by (3.4) are inside $\Gamma_{e}, \arg F(z)$ increases as $t$ traverses $\Gamma_{e}$ in the positive sense and so $F(z)$ is starlike. Hence a polynomial is always convex on every ellipse enclosing all of its zeros. Note that for an $n$-th degree polynomial the image curve will go around the origin $n$ times, and so the "region" enclosed will not be simple, and of course not convex in the ordinary sense, but the boundary curve will be convex in the sense of our definition.

In the next section we shall prove the following more general assertion.

Theorem 3.1. Let $p(z)$ be a polynomial and let $\Gamma$ be the boundary of any simple convex region containing all the zeros of $p(z)$. Then $p(z)$ is convex on $\Gamma$.

Theorem 3.1, though not explicitly proved, is suggested by Alexander [1], however our approach is different.

4. The family of parallel lines. Let $\Gamma_{v}$ denote the set of vertical lines with positive direction upward. We set $z=u+i t$, and then the conditions (2.2) and (2.4) for convexity and starlikeness become respectively

$$
\mathfrak{C}\left(\Gamma_{v}, f, t\right)=\mathbb{R} \frac{f^{\prime \prime}(z)}{f^{\prime}(z)} \geq 0
$$


and

$$
\varsigma\left(\Gamma_{v}, F, t\right)=R \frac{F^{\prime}(z)}{F(z)} \geq 0 .
$$

From Theorem 2.3 we have immediately

Theorem 4.1. If in a region $R$,

$$
F(z)=h f^{\prime}(z),
$$

where $h$ is some complex constant, then $f(z)$ convex on the family $\Gamma_{v}$ implies $F(z)$ starlike on $\Gamma_{v}$, and conversely.

TheOREM 4.2. Let

$$
F(z)=1+\sum_{n=1}^{\infty} A_{n} z^{n} \quad\left(\alpha_{1}=\left|A_{1}\right|\right),
$$

be regular and not zero in $|z|<1$. If $F(z)$ is starlike on $\Gamma_{v}$ in $|z|<1$, then for $n=1,2,3, \cdots$

$$
\left|A_{n}\right| \leq \sum_{k=0}^{n} \frac{\left(-\alpha_{1}\right)^{n-k} 2 \alpha_{1}\left(2 \alpha_{1}+1\right) \cdots\left(2 \alpha_{1}+k-1\right)}{(n-k) ! k !}
$$

and if $A_{1}=\alpha_{1} \geq 0$ this inequality is sharp for each positive integer $n$, as shown by the function

$$
F^{*}(z)=e^{-\alpha_{1} z}(1-z)^{-2 \alpha_{1}} .
$$

Proof. From equation (4.2) we have for

$$
G(z)=\frac{F^{\prime}(z)}{F(z)}=c_{0}+c_{1} z+\cdots,
$$

$\mathscr{R}(G(z)) \geq 0$ in $|z|<1$, and hence by a theorem due to Carathéodory [2], $\left|c_{n}\right| \leq 2 \Re\left(c_{0}\right)=2 \Re\left(A_{1}\right) \leq 2 \alpha_{1}, n=1,2, \cdots$. Thus

$$
\frac{F^{\prime}(z)}{F(z)}<<\alpha_{1}+2 \sum_{n=1}^{\infty} \alpha_{1} z^{n}=\alpha_{1} \frac{1+z}{1-z}
$$

where the notation $<<$ means that for the two power series in (4.6) the coefficients on the right hand side are real and not less than the absolute value of the corresponding coefficients on the left hand side [3;9]. By integration

$$
\begin{gathered}
\log F(z)<<-2 \alpha_{1} \log (1-z)-\alpha_{1} z \\
F(z)<<\frac{1}{e^{\alpha_{1} z}(1-z)^{2 \alpha_{1}}}=F^{*}(z) .
\end{gathered}
$$

The coefficients in the power series for $F^{*}(z)$ are given by the right side of (4.7). It is easy to see that the bound is sharp since $R\left(F^{* \prime}(z) / F^{*}(z)\right)>0$ in $|z|<1$.

Combining Theorems 4.2 and 4.1 with $h=1$ yields immediately 
THEOREM 4.3. Let

$$
f(z)=z+\sum_{n=2}^{\infty} a_{n} z^{n} \quad\left(\alpha_{2}=\left|a_{2}\right|\right)
$$

be regular and $f^{\prime}(z) \neq 0$ in $|z|<1$. If $f(z)$ is convex on the family $\Gamma_{v}$ in $|z|<1$, then for $n=2,3, \cdots$

$$
\left|a_{n}\right| \leq \frac{1}{n} \sum_{k=0}^{n-1} \frac{\left(-2 \alpha_{2}\right)^{n-k-1} 4 \alpha_{2}\left(4 \alpha_{2}+1\right) \cdots\left(4 \alpha_{2}+k-1\right)}{(n-k-1) ! k !}
$$

and if $a_{2}=\alpha_{2} \geq 0$ this inequality is sharp for $n=2,3, \cdots$ as the function

$$
f^{*}(z)=\int_{0}^{z} \frac{e^{-2 \alpha_{2} \zeta}}{(1-\zeta)^{4 \alpha_{2}}} d \zeta
$$

shows. In particular if $a_{2}=0$ then $f(z)=z$.

We now prove Theorem 3.1. First assume $p(z)$ is a polynomial with zeros $a_{k}$ such that $R a_{k}<0$ for $k=1,2, \cdots, n$. Then the zeros of $p^{\prime}(z), b_{k}$, lie in the same half-plane. Hence

$$
\Re \frac{p^{\prime \prime}(z)}{p^{\prime}(z)}=\Re\left(\sum_{k=1}^{n-1} \frac{1}{z-b_{k}}\right)>0
$$

for $z$ on the imaginary axis, and so by (4.1), $p(z)$ is convex on this axis. By a rotation, it is obvious that $p(z)$ is convex on any directed line with the property that all the zeros of $p(z)$ lie to the left of the directed line. The proof is completed by noting that the boundary of any convex region can be approximated by convex polygons.

Let $\Gamma_{\beta}, 0 \leq \beta<\pi$, be the family of parallel lines cutting the positive real axis at an angle $\beta$, the positive direction of the lines being the direction of increasing $y$. In particular $\Gamma_{\pi / 2}=\Gamma_{v}$. We set $z=u+t \cos \beta+i t \sin \beta$, and then the conditions (2.2) and (2.4) for convexity and starlikeness become respectively

$$
\mathcal{C}\left(\Gamma_{\beta}, f, t\right)=\Re\left(-i e^{i \beta} \frac{f^{\prime \prime}(z)}{f^{\prime}(z)}\right) \geq 0
$$

and

$$
\S\left(\Gamma_{\beta}, F, t\right)=a\left(-i e^{i \beta} \frac{F^{\prime}(z)}{F(z)}\right) \geq 0 .
$$

Suppose now that $f(z)$ is simultaneously convex in $R$ on the two families, $\Gamma_{\beta}$ and $\Gamma_{\delta}, 0<\beta<\delta<\pi$. Observe that the condition $R\left(-i e^{i \beta} g(z)\right)>0$ for a region $R$ is equivalent to the statement that $g(z)$ assumes in $R$ only values in the half-plane to the right of the directed line $w=t e^{i(x-\beta)}$. If the values of $g(z)$ in $R$ lie to the right of two directed lines $w_{1}=t e^{i(\pi-\beta)}$ and $w_{2}=t e^{i(\pi-\delta)}$, 
$0<\beta<\delta<\pi$, then the values of $g(z)$ lie to the right of any directed line $w=t e^{i(\pi-\theta)}$ with $\beta \leq \theta \leq \delta$. This gives

TheOREM 4.4. If $f(z)$ is regular in a region $R$ and is convex simultaneously on the straight lines $\Gamma_{\beta}$ and $\Gamma_{\delta}, 0<\beta<\delta<\pi$, then for each $\theta, \beta \leq \theta \leq \delta, f(z)$ is convex on the lines $\Gamma_{\theta}$.

This theorem and the proof remain valid when the word "convex" is replaced by "starlike," but in this latter case the proof can be given by a trivial geometric argument, and the mapping function $f(z)$ does not even need to be regular.

5. Pencils of lines. Let $\Gamma_{P}(a), a$ real, denote the pencil of lines passing through the point $i a$, the positive direction being the direction of increasing $y$. We set $z=i a+e^{i u} t=t \cos u+i(a+t \sin u), 0<u<\pi$. The conditions (2.2) and (2.4) for convexity and starlikeness become respectively

$$
\mathfrak{C}\left(\Gamma_{P}(a), f, t\right)=-\Re\left(\frac{f^{\prime \prime}(z)}{f^{\prime}(z)} \frac{a+i z}{t}\right) \geq 0,
$$

and

$$
\S\left(\Gamma_{P}(a), F, t\right)=-\Re\left(\frac{F^{\prime}(z)}{F(z)} \frac{a+i z}{t}\right) \geq 0,
$$

for $t \neq 0$.

Let us suppose first $a=0$. Then both (5.1) and (5.2) have the general form $\mathscr{I}(g(z) / t) \geq 0$, with appropriate definition for $g(z)$. But below the real axis $t<0$, and above the real axis $t>0$, so that $g(g(z))$ is either zero or has the sign of $\mathscr{I}(z)$ off of the real axis, and by continuity must be zero on the real axis. If at one point off of the real axis $\mathscr{g}(g(z))=0$ then $\mathscr{T}(g(z)) \equiv 0$ and $g(z)$ is a constant. Functions with the property that $\mathscr{g}(g(z))$ has the same sign as $\mathscr{G}(z)$ have been named typically-real by Rogosinski [4]. This proves

Theorem 5.1. Let $f(z)$ be regular and $f^{\prime}(z) \neq 0$ in a region $R$ containing a segment of the real axis. Then a necessary and sufficient condition that $f(z)$ be convex on the pencil of lines $\Gamma_{P}(0)$ is that

$$
g(z)=\frac{z f^{\prime \prime}(z)}{f^{\prime}(z)}
$$

is either typically-real in $R$, or identically zero. Further if $F(z)$ is regular and not zero in such a region $R$ then a necessary and sufficient condition that $F(z)$ be starlike on the pencil $\Gamma_{P}(0)$ is that

$$
G(z)=\frac{z F^{\prime}(z)}{F(z)}
$$

is either typically-real in $R$, or identically zero. 
The bounds for the power series coefficients of functions, typically-real in $|z|<1$, are well known. An integration gives bounds for the power series coefficients of $f(z)$ and $F(z)$, namely,

Theorem 5.2. Let $f(z)$ given by $(4.8)$ be regular, $f^{\prime}(z) \neq 0$ in $|z|<1$, and $f(z)$ convex on the pencil $\Gamma_{P}(0)$. Let $F(z)$ given by $(4.4)$ be regular, $F(z) \neq 0$, in $|z|$ $<1$, and $F(z)$ starlike on the pencil $\Gamma_{P}(0)$. Then

$$
\begin{array}{ll}
\left|a_{n}\right| \leq\left|b_{n}\right| & (n=3,4,5, \cdots), \\
\left|A_{n}\right| \leq\left|B_{n}\right| & (n=2,3,4, \cdots),
\end{array}
$$

where $b_{n}$ and $B_{n}$ are defined by

$$
f_{P}^{*}(z)=\int_{0}^{z} e^{2\left|a_{2}\right| \zeta /(1-\zeta)} d \zeta=\sum_{n=1}^{\infty} b_{n} z^{n}
$$

and

$$
F_{P}^{*}(z)=e^{\left|A_{1}\right| z /(1-z)}=\sum_{n=0}^{\infty} B_{n} z^{n} .
$$

If $A_{1}>0$ and $a_{2}>0$, the inequalities (5.5) and (5.6) are sharp, since $f_{P}^{*}(z)$ and $F_{P}^{*}(z)$ are respectively convex and starlike on the lines $\Gamma_{P}(0)$.

The proof is similar to that of Theorem 4.2 so we omit the details.

Let us consider the lines of $\Gamma_{P}(a)$ and $\Gamma_{P}(b)$ which lie in the unit circle. If $b>a \geq 1$, then $t$ is negative and the condition for convexity (5.1) can be written.

$$
\begin{aligned}
& \propto \frac{f^{\prime \prime}(z)}{f^{\prime}(z)}(a+i z) \geq 0, \\
& \propto \frac{f^{\prime \prime}(z)}{f^{\prime}(z)}(b+i z) \geq 0,
\end{aligned}
$$

from which, if $\mu>0, \nu>0$ and $\mu+\nu=1$ we find

$$
\mathcal{Q} \frac{f^{\prime \prime}(z)}{f^{\prime}(z)}(\mu a+\nu b+i z) \geq 0 \text {. }
$$

Hence if $1 \leq a \leq c \leq b$, convexity on $\Gamma_{P}(a)$ and $\Gamma_{P}(b)$ implies convexity on $\Gamma_{P}(c)$.

For convexity in $|z|<1$ on $\Gamma_{P}(-b), b \geq 1, t$ is positive, and (5.1) becomes

$$
\Re \frac{f^{\prime \prime}(z)}{f^{\prime}(z)}(b-i z) \geq 0 \text {. }
$$

If $\mu>\nu>0$ we deduce from (5.9) and (5.12)

$$
\mathfrak{R} \frac{f^{\prime \prime}(z)}{f^{\prime}(z)}\left(\frac{\mu a+\nu b}{\mu-\nu}+i z\right) \geq 0,
$$


and if $\nu>\mu>0$

$$
\mathcal{Q} \frac{f^{\prime \prime}(z)}{f^{\prime}(z)}\left(\frac{\mu a+\nu b}{\nu-\mu}-i z\right) \geq 0
$$

We have proved

Theorem 5.3. Let $f(z)$ be regular, $f^{\prime}(z) \neq 0$ in $|z|<1$. (1) If $f(z)$ is simullaneously convex on the lines $\Gamma_{P}(a)$ and $\Gamma_{P}(b), b>c>a \geq 1$, then $f(z)$ is convex on the lines $\Gamma_{P}(c)$. (2) If $f(z)$ is simultaneously convex on the lines $\Gamma_{P}(a), \Gamma_{P}(-b)$, $a, b \geq 1$, and if $c>a$ or $c<-b$, then $f(z)$ is convex on the lines $\Gamma_{P}(c)$.

In particular, if $c=\infty, f(z)$ is convex on the lines $\Gamma_{v}$.

A similar theorem is valid for starlikeness, but in this case the proof can be given by a purely geometric argument. It should be obvious that in Theorem 5.3 the circle $|z|<1$ can be replaced by any region which lies in a strip parallel to the real axis if the strip does not contain any of the points $a i, b i$, or $-b i$.

From inequalities of the type (5.9) it is possible to obtain bounds for $|f(z)|$, $|\arg f(z)|$, and the coefficients for functions convex (starlike) on $\Gamma_{P}(a)$ in $|z|$ $<1$. However the bounds which we have obtained in this case do not appear to be sharp.

6. The family of logarithmic spirals. Let $\Gamma_{s}$ denote the family of logarithmic spirals

$$
z=u e^{-i k t}+c .
$$

Then $z^{\prime}(t)=-i k(z-c)$ and in this case Theorem 2.3 states that if for some constant $h$

$$
F(z)=h f^{\prime}(z)(z-c),
$$

then $f(z)$ convex on $\Gamma_{S}$ implies $F(z)$ starlike on $\Gamma_{S}$, and conversely.

Next suppose that $F(z)$ given by equation (4.4) is regular and $F(z) \neq 0$ in $|z|<1$. The condition of Theorem 2.2 for starlikeness on $\Gamma_{S}$ yields

$$
\Re \frac{F^{\prime \prime}(z)}{F(z)} k(c-z) \equiv R G(z) \geq 0 .
$$

Since $G(0)=A_{1} k c$, we have

$$
\frac{F^{\prime}(z)}{F(z)} k(c-z)<<\left|A_{1} k c\right| \frac{1+z}{1-z}
$$

and if $c \geq 1$,

$$
\frac{F^{\prime}(z)}{F(z)}<<\left|A_{1}\right| \frac{1+z}{1-z} \cdot \frac{1}{1-z / c}
$$


On integration, with $c>1$,

$$
F(z)<<F_{S}^{*}(z) \equiv\left[\frac{(1-z / c)^{1+c}}{(1-z)^{2}}\right]^{c \mid A_{1} / / c-1} .
$$

In case $c=1,(6.6)$ is replaced by

$$
F(z)<<F_{S}^{*}(z) \equiv(1-z)^{\left|A_{1}\right|} e^{2\left|A_{1}\right| z /(1-z)} .
$$

Theorem 6.1. If $F(z)$ given by equation (4.4) is regular, $F(z) \neq 0$ in $|z|<1$, and if $F(z)$ is starlike on the family of spirals $\Gamma_{S}, c \geq 1$, then

$$
\left|A_{n}\right| \leq\left|B_{n}\right| \quad(n=2,3, \cdots),
$$

where $B_{n}$ is defined by

$$
F_{S}^{*}(z)=1+\sum_{n=1}^{\infty} B_{n} z^{n}
$$

and $F_{S}^{*}(z)$ is given by (6.6) if $c>1$, and (6.7) if $c=1$. If $k>0$ and $A_{1}>0$ (the spirals become circles) the inequality (6.8) is sharp for every $n$, since the functions $F_{S}^{*}(z)$ are starlike on $\Gamma_{S}$.

From this theorem, and equation (6.1) with $h=-1 / c$, a similar theorem can be obtained for functions convex on $\Gamma_{S}$.

7. Circles. If in $\$ 6, k$ is real and negative, the spirals become circles with center $c$, the positive direction being counterclockwise. Let $\Gamma(c, r)$ denote such a circle of radius $r$ and center $c$. The conditions (2.2) and (2.4) for convexity and starlikeness on $\Gamma(c, r)$ become respectively

$$
1+\Re \frac{f^{\prime \prime}(z)}{f^{\prime}(z)}(z-c) \geq 0,
$$

and

$$
\text { Q } \frac{F^{\prime}(z)}{F(z)}(z-c) \geq 0 \text {. }
$$

Combining these equations for $c=0$, with the equations (4.1) and (4.2) for $\Gamma_{v}$ we have immediately

THeOREM 7.1. If in a region $R, f(z)$ is convex on the circles $\Gamma(0, r)$ and on the vertical lines $\Gamma_{v}$, then for every $c<0, f(z)$ is convex on the circles $\Gamma(c, r)$. If $f(z)$ is convex on the circles $\Gamma\left(c_{1}, r\right)$ and $\Gamma\left(c_{2}, r\right)$ with $c_{1}<c_{2}$, then for each $c, c_{1} \leq$ $c \leq c_{2}, f(z)$ is convex on the circles $\Gamma(c, r)$.

8. Non-analytic functions. In this section we indicate an interesting distinction between convexity and starlikeness, which comes to light only when mappings by non-analytic functions are considered. We shall investigate only the possibility of generalizing Theorem 4.4. 
Suppose $f(z)=u(x, y)+i v(x, y)$, where $u$ and $v$ are two real functions of the real variables $x$ and $y$ which possess continuous second order partial derivatives. As in $\S 2$ let $\Gamma$ be a directed curve $z=z(t)=x(t)+i y(t)$. Then with the same notation as in $\S 2$, a short computation gives the following result.

If on $\Gamma$,

$$
J\left(\frac{u, v}{x, y}\right) \neq 0 \quad \text { and } \quad z^{\prime}(t) \neq 0
$$

then a necessary and sufficient condition that $f(z)$ be convex on $\Gamma$ is that on $\Gamma$

$$
\begin{aligned}
\mathcal{C}(\Gamma, f, t) \equiv\left(\frac{d x}{d t}\right)^{3}\left(u_{x} v_{x x}-\right. & \left.v_{x} u_{x x}\right) \\
& +\left(\frac{d x}{d t}\right)^{2}\left(\frac{d y}{d t}\right)\left(2 u_{x} v_{x y}-2 v_{x} u_{x y}+u_{y} v_{x x}-v_{y} u_{x x}\right) \\
& +\left(\frac{d x}{d t}\right)\left(\frac{d y}{d t}\right)^{2}\left(2 u_{y} v_{x y}-2 v_{y} u_{x y}+u_{x} v_{y y}-v_{x} u_{y y}\right) \\
& +\left(\frac{d y}{d t}\right)^{3}\left(u_{y} v_{y y}-v_{y} u_{y y}\right) \\
& +\left(\frac{d x}{d t} \frac{d^{2} y}{d t^{2}}-\frac{d y}{d t} \frac{d^{2} x}{d t^{2}}\right)\left(u_{x} v_{y}-v_{x} u_{y}\right) \geq 0 .
\end{aligned}
$$

If $F(z)=U(x, y)+i V(x, y) \neq 0$ on $\Gamma$ then a necessary and sufficient condition for starlikeness on $\Gamma$ is that on $\Gamma$

$$
\mathcal{S}(\Gamma, F, t) \equiv\left(U V_{x}-V U_{x}\right) \frac{d x}{d t}+\left(U V_{y}-V U_{y}\right) \frac{d y}{d t} \geq 0 .
$$

For the family of straight lines $\Gamma_{\beta}$ of $\S 4$, conditions (8.1) and (8.2) become respectively

$$
\begin{gathered}
\cos ^{3} \beta\left(u_{x} v_{x x}-v_{x} u_{x x}\right)+\cos ^{2} \beta \sin \beta\left(2 u_{x} v_{x y}-2 v_{x} u_{x y}+u_{y} v_{x x}-v_{y} u_{x x}\right) \\
+\cos \beta \sin ^{2} \beta\left(2 u_{y} v_{x y}-2 v_{y} u_{x y}+u_{x} v_{y y}-v_{x} u_{y y}\right) \\
+\sin ^{3} \beta\left(u_{y} v_{y y}-v_{y} u_{y y}\right) \geq 0
\end{gathered}
$$

and

$$
\left(U V_{x}-V U_{x}\right) \cos \beta+\left(U V_{y}-V U_{y}\right) \sin \beta \geq 0 \text {. }
$$

It is easy to see from equation (8.4) that Theorem 4.4 generalizes for starlikeness of non-analytic functions, namely,

THEOREM 8.1. If $F(z)$ is starlike in $R$ on the lines $\Gamma_{\delta}$ and the lines $\Gamma_{\beta}, 0<$ $\beta<\delta<\pi$, then $F(z)$ is starlike on the lines $\Gamma_{\theta}$ for $\beta \leq \theta \leq \delta$. 
Proof. Setting $U V_{x}-V U_{x}=A, U V_{y}-V U_{y}=B$ it is easy to see that for fixed $x, y$ the function

$$
X(\theta)=A \cos \theta+B \sin \theta
$$

has simple zeros at intervals of length $\pi$. Hence if $X(\beta) \geq 0$ and $X(\delta) \geq 0$, $0<\delta-\beta<\pi$, it follows that $X(\theta) \geq 0$ for $\beta \leq \theta \leq \delta$.

Next consider the convexity of the example function

$$
f(z)=2 x y+y^{2}+2 x+y+i\left(2 x y+y^{2}+2 y\right),
$$

for which $J(u, v / x, y)=2(2 x+3 y+2)$. For this function, the condition (8.3) becomes

$$
Q(\beta)=8 \cos ^{2} \beta \sin \beta-2 \sin ^{3} \beta \geq 0 .
$$

Now $Q(\pi / 2)<0, Q(3 \pi / 4)>0$ and $Q(\pi / 4)>0$. Thus for any region not intersecting the line $2 x+3 y+2=0$, the function $f(z)$ given by (8.6) is simultaneously convex on the lines $\Gamma_{3 \pi / 4}$ and $\Gamma_{\pi / 4}$, but is not convex on the lines $\Gamma_{\pi / 2}$.

\section{REFerences}

1. J. W. AleXander, II, Functions which map the interior of the unit circle upon simple regions, Annals of Mathematics (2), vol. 17 (1915-16), pp. 12-22.

2. C. Carathédony, Über den Variabilitätsbereich der Fourier'schen Konstanten von positiven harmonischen Funktionen, Rendiconti del Circolo Matematico di Palermo, vol. 32(1911), pp. 193-217.

3. G. Pólya and G. Szegö, Aufgaben und Lehrsätze, vol. I, New York, 1945.

4. Werner Rogosinski, Über positive harmonische Entwicklungen und typisch-reele potenzreihen, Mathematische Zeitschrift, vol. 35(1932), pp. 93-121.

UNIVERSITY OF KeNTUCKY. 